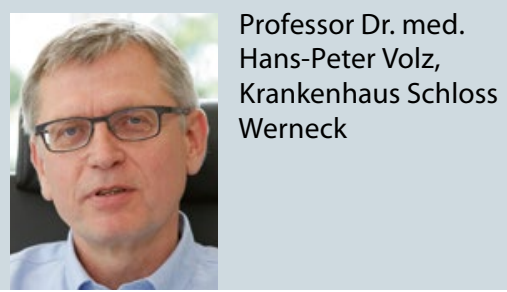

\title{
Neue Studien zur Schizophrenie
}

\author{
Der Studienüberblick gibt Antworten auf die Fragen, wie lange bei schizophrenen Patienten eine anti- \\ psychotische Medikation bei einem initial ausbleibenden Therapieerfolg fortgeführt werden sollte, \\ wie sich die primäre Negativsymptomatik behandeln lässt und welche Patienten im besonderen Maße \\ von Depot-Antipsychotika profitieren. Zwei weitere Arbeiten beschäftigen sich mit der Ätiopathogenese \\ hinsichtlich kognitiver Leistungseinbußen und den Ursachen für hirnstrukturelle Veränderungen.
}

\section{Antipsychotikatherapie: In Woche 2 entscheidet sich der Langzeiterfolg}

Eine Intervention, die nach zwei Wochen nicht eine mindestens $20 \%$ ige Verbesserung erzielt, hat eine geringe Chance auf langfristigen Response, so das Ergebnis einer Metaanalyse.

E ine ungeklärte Frage im Rahmen - des Beginns einer antipsychotischen Medikation bei schizophrenen Patienten ist, wie lange diese bei einem initial aus-

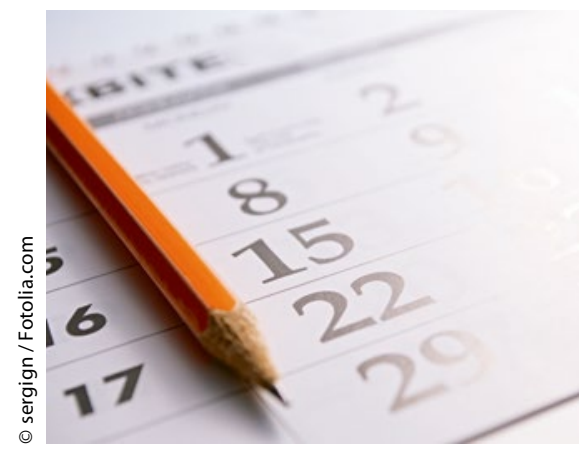

Deutet sich nach zwei Wochen kein Therapieerfolg an, ist ein Wechsel bereits angebracht. bleibenden Therapieerfolg weiter fortgeführt oder wann ein Wechsel vorgenommen werden sollte.

Dieser Frage ging eine Metaanalyse nach. Als wirksam wurde eine Intervention eingestuft, wenn sie zum Ende der Studie (Studienlängen variierten von vier bis zwölf Wochen) einen Abfall von mindestens $50 \%$ auf der Positive and Negative Syndrome Scale (PANSS) beziehungsweise Brief Psychiatric Rating Scale (BPRS) generiert hatte. Als Prädiktionsmaß wurde eine Grenze von $20 \%$ Verbesserung zu Woche 2 bestimmt. Das bedeutete: Falls Patienten sich nicht um mindestens $20 \%$ zu Woche 2 auf einem der beiden Psychopathologiemaße verbessert hatten, wurde vorausgesagt, dass auch am Ende der Studie kein Erfolg eintreten wird, beziehungsweise, falls sie sich um mindestens $20 \%$ zu Woche 2 gebessert hatten, würden sie auch eine Response (mindestens 50\%ige Abnahme zum Studienendpunkt) erreichen.

Es wurden 34 Studien identifiziert, aus denen die genannten Parameter generiert werden konnten. Eine weniger als $20 \%$ ige PANSS- oder BPRS-Reduktion zu Woche 2 sagte eine Nonresponse zum Studienendpunkt mit einer Spezifität von $86 \%$ und einem positiven prädiktiven Wert (PPW) von $90 \%$ voraus.

\section{Kommentar}

Diese Studienergebnisse sollten verstärkt in die klinische Praxis Einzug halten, um die hohe Anzahl langer Therapieversuche, die letztendlich dann größtenteils doch nicht den erstrebten Erfolg zeitigen, zu verhindern. Zeigt sich nach zwei Wochen keine oder kaum eine Besserung sollte eine deutliche Modifikation/Wechsel der pharmakologischen Intervention erfolgen.

Prof. Dr. med. Hans-Peter Volz

Samara MT et al. Early improvement as a predictor of later response to antipsychotics in schizophrenia: a diagnostic test review. Am J Psychiatry $2015 ; 172: 617-29$ 